\title{
The Empirical Research of the IFDI's Influence upon the Comparative Advantage of Export Products from China
}

\author{
Ming Yang ${ }^{1}$, Hong-mei Chang ${ }^{2}$ \\ ${ }^{1)}$ School of Law and Politics, Tianjin University of Technology, Tianjin, China \\ ${ }^{2}$ School of Law and Politics, Tianjin University of Technology, Tianjin, China
}

\begin{abstract}
The comparative advantage index of export products from China according to the technology intensity classification shows that low technology products are still the main source of China's comparative advantage, but their level is going through a decreasing process. The level of the comparative advantage of high technology products is on an increasing trend. Using the data during 1992-2012, this paper's empirical test shows that IFDI has played a positive role in China's comparative advantage transition from low technology products to high technology products by promoting technical progress, shifting the endowment structure of production factors. So China should continue to attract actively foreign direct investment, especially to guide the multinational companies to invest in high technology industries and promote IFDI's spillover and industry correlation effect.
\end{abstract}

Keywords-IFDI , Export commodities, Comparative advantage.

\section{IFDI 对中国出口产品比较优势变动的实证研究}

\author{
杨明常宏梅 \\ 天津理工大学法政学院, 天津, 中国
}

\begin{abstract}
摘 要 以技术密集度分类的中国出口品比较优势指数显示, 低技术商品仍是中国比较优势的主要来源, 但其水平呈现出递减的 过程, 而中高技术商品比较优势水平呈递增趋势。论文基于 1992-2012 年数据实证检验, IFDI 通过促进技术进步、要素禀赋结构转变 等对中国出口产品比较优势由低向高过渡起到了积极作用。中国应继续积极吸引外商直接投资, 特别是引导跨国公司投资于高新技术 产业，促进 IFDI 的溢出和产业关联效应。
\end{abstract}

关键词 IFDI, 出口商品, 比较优势

\section{1. 引言}

在中国巨额出口中, 输入性外商直接投资 (IFDI) 占 有较大比重。自 2001 年始, 外资企业出口额占到了中国出 口的 50\%以上。面临 “中国模式” 的重要问题是： IFDI 对 中国比较优势产品的升级是否起到了积极作用?

针对 IFDI 对中国比较优势产品结构变动的影响, 蒋殿 春、张宇 (2006) 实证研究证明, 外商直接投资推进了相 关行业的技术进步, 而且在高技术行业中得到了最明显的 体现。冯梅 (2012) 实证研究的中国制造业 RCA 值与外商 直接投资 (三资工业企业总产值) 指标呈正相关关系。论 文基于对中国出口产品的比较优势结构情况的分析, 利用 1992-2012 年度数据, 实证检验了 IFDI 对中国比较优势产 品结构变动的作用, 提出 FDI 吸收的政策建议。

\section{2. 中国分类出口商品比较优势的变动}

对产品比较优势的测算有多种指标, 其中运用显示性 比较优势指数最为普遍。按 Balassa (1981) 给出的显示性 比较优势指数 (Revealed Comparative Advantage, RCA) 计算方法, RCA 等于一国某种商品占本国总出口值的比重 与世界这一比重的比较, $0<\mathrm{RCA}<\infty$, RCA 的值越大, 比 较优势程度越高。当 $\mathrm{RCA}<1$ 时, 缺乏比较优势, 当 $\mathrm{RCA}>1$ 时, 具有比较优势, 当 $R C A>2.5$ 时, 反映该国在该产品上 具有很强的比较优势。对商品分类的一种方法是按商品要 素密集度分类。一般来讲, 资源密集型、劳动密集型商品 的生产复杂程度较低, 商品的价格弹性较高, 获得的贸易 利益较少; 资本密集型商品、特别是人力资本密集型商品 生产复杂程度高, 获得的贸易利益较多。如郑展鹏（2010） 
将出口商品分为自然资源密集型商品、非熟练劳动密集型 商品、资本密集型商品、人力资本密集型商品四类来分析 中国对外贸易结构及出口竞争力。但这种分类法忽视了技 术密集度在商品贸易利益中的作用。近年来很多研究倾向 于按照商品的技术密集度进行分类。低技术密集型商品需 求的价格弹性较高, 获得的贸易利益较少; 而高技术密集
型商品生产复杂, 获得的贸易利益较多。如 Worz(2005)就 是按照技术密集度把贸易品分成高技术密集商品、中技术 密集商品和低技术密集商品三类。Lall(2004)在对发展中国 家制造业出口结构进行研究时, 将 SITC 三位数分类的商品 分为初级商品 $(\mathrm{PP})$ 、资源型商品 $(\mathrm{RB})$ 、低技术商品 (LT)、中技术商品(MT)和高技术商品（HT）。

表 1 中国按技术密集度分类出口商品的显示性比较优势指数

\begin{tabular}{|c|c|c|c|c|c|c|c|c|c|c|}
\hline 年度 & $R C A_{P P_{t}}$ & $R C A_{R B_{1 t}}$ & $R C A_{R B_{2 t}}$ & $R C A_{L T_{1 t}}$ & $R C A_{L T_{2 t}}$ & $R C A_{M T_{1 t}}$ & $R C A_{M T_{2 t}}$ & $R C A_{M T_{3 t}}$ & $R C A_{H T_{t}}$ & $R C A_{H T_{2}}$ \\
\hline 1992 & 1.27 & 0.71 & 0.71 & 4.09 & 1.51 & 0.09 & 0.89 & 0.60 & 0.54 & 0.48 \\
\hline 1993 & 1.22 & 0.77 & 0.62 & 4.23 & 1.41 & 0.11 & 0.82 & 0.61 & 0.59 & 0.47 \\
\hline 1994 & 1.05 & 0.72 & 0.65 & 4.15 & 1.08 & 0.10 & 0.74 & 0.65 & 0.62 & 0.49 \\
\hline 1995 & 0.83 & 0.76 & 0.83 & 3.94 & 0.87 & 0.11 & 1.12 & 0.68 & 0.72 & 0.59 \\
\hline 1996 & 0.76 & 0.81 & 0.77 & 3.93 & 0.89 & 0.11 & 0.97 & 0.72 & 0.85 & 0.59 \\
\hline 1997 & 0.82 & 0.70 & 0.77 & 3.88 & 0.74 & 0.11 & 1.07 & 0.71 & 0.85 & 0.56 \\
\hline 1998 & 0.77 & 0.63 & 0.76 & 3.80 & 0.72 & 0.11 & 0.97 & 0.74 & 0.99 & 0.56 \\
\hline 1999 & 0.67 & 0.64 & 0.70 & 3.80 & 0.70 & 0.12 & 0.95 & 0.80 & 1.06 & 0.58 \\
\hline 2000 & 0.58 & 0.69 & 0.65 & 3.80 & 0.59 & 0.18 & 1.12 & 0.83 & 1.13 & 0.60 \\
\hline 2001 & 0.51 & 0.68 & 0.68 & 3.57 & 0.54 & 0.17 & 0.96 & 0.87 & 1.33 & 0.49 \\
\hline 2002 & 0.47 & 0.64 & 0.63 & 3.41 & 0.44 & 0.17 & 0.81 & 0.91 & 1.56 & 0.47 \\
\hline 2003 & 0.43 & 0.57 & 0.61 & 3.20 & 0.33 & 0.18 & 0.85 & 0.92 & 1.81 & 0.56 \\
\hline 2004 & 0.35 & 0.58 & 0.59 & 3.13 & 0.24 & 0.20 & 0.97 & 0.95 & 1.94 & 0.64 \\
\hline 2005 & 0.31 & 0.60 & 0.54 & 3.12 & 0.18 & 0.22 & 0.93 & 1.00 & 1.93 & 0.75 \\
\hline 2006 & 0.28 & 0.65 & 0.49 & 3.21 & 0.15 & 0.25 & 0.88 & 1.04 & 2.06 & 0.69 \\
\hline 2007 & 0.26 & 0.61 & 0.49 & 3.12 & 0.11 & 0.29 & 0.87 & 0.97 & 2.20 & 0.77 \\
\hline 2008 & 0.21 & 0.58 & 0.74 & 3.25 & 0.10 & 0.33 & 0.84 & 1.13 & 2.31 & 0.82 \\
\hline 2009 & 0.25 & 0.56 & 0.65 & 3.20 & 0.12 & 0.32 & 0.67 & 1.21 & 2.24 & 0.82 \\
\hline 2010 & 0.25 & 0.57 & 0.64 & 4.20 & 0.09 & 0.31 & 0.80 & 1.18 & 2.21 & 0.86 \\
\hline 2011 & 0.33 & 0.64 & 0.70 & 3.15 & 0.08 & 0.32 & 0.86 & 1.10 & 2.13 & 0.78 \\
\hline 2012 & 0.32 & 0.62 & 0.71 & 3.13 & 0.08 & 0.32 & 0.85 & 1.12 & 2.14 & 0.76 \\
\hline
\end{tabular}

数据来源:根据联合国贸发会议网站http://comtrade.un.org/db/数据计算。

我们按照 Lall 的分类方法计算了中国各类出口商品的 比较优势, 结果显示: 改革开放之初, 中国比较优势产品 以 $P P$ 和 RB 以及 $L T$ 为主, 但中国靠 PP 和 RB 带动对外出 口的状况迅速改变, 这两类出口品的比较优势水平不断下 降, 进入到 20 世纪 90 年代, 比较优势指数降低到 1 以下; LT 具有最强比较优势地位, 其中 LT1 的比较优势最强, 到 2012 年, 比较优势指数仍然大于 3 , 但 LT 的比较优势水平 一直处于递减状态; MT 和 HT 的比较优势在 2001 年之前 处于比较劣势, 但却呈不断上升态势, 其中 MT3 在 2005 年之后取得比较优势 $(\mathrm{RCA}>1)$; 高技术产品在 2002 年之 后获得比较优势, 其中 HT1 2000 年之后成为具有比较优势
的产品, 且比较优势越来越明显, 到 2012 年 HT1 的 RCA 上升到 2.14。（见表 1)

\section{IFDI 对比较优势的影响路径}

一国比较优势结构决定于该国的技术进步和要素禀赋 结构的转变, 而 IFDI 能够通过影响其决定因素对东道国比 较优势产品结构产生实质性影响。

\subsection{IFDI 与东道国的技术进步}

技术进步主要来源于技术创新和对技术扩散的吸收, IFDI 技术扩散主要通过资本存量效应、边干边学、竞争效 
应、人员培训与流动等多种路径实现。根据内生增长理论, 来自于边干边学的技术进步是东道国资本存量的函数, 如 果 IFDI 对本国资本引致效应大于挤出效应, 则 IFDI 有利 于中国资本存量的提高, 促进技术进步。在技术创新方面, 在一个资本存量较多和技术水平较高的经济体中, 研发部 门的技术创新率较高, 外资的进入为其提供了外生资本和 技术, 有利于东道国技术创新的提高。而且外资企业的进 入增加了产品市场竞争压力, 带来竞争效应, 迫使东道国 厂商加速生产技术和质量更高的产品。

\subsection{IFDI 对东道国要素禀赋结构变动的影响}

从中国外商直接投资吸收的实际历程来看, 外商直接 投资首先进入了中国的劳动密集型产业, 具有直接的资本 效应和比较优势的发挥。在经历了外商直接投资大规模进 入劳动密集型产业之后, 到 20 世纪 90 年代, 外商直接投 资也开始大量进入中国的资本、技术密集型产业。外商直 接投资的资本效应还表现在将资产存量由低质量变成高质 量, 或者新增高质量资产, 有利于从根本上改变比较优势 结构形成的物质基础。IFDI 还有利于人力资本的积累, 当 新技术被跨国公司引入后, 不仅自身需要高技能劳动力, 而且也会促使其他企业进行技术创新, 提高技术密集程度, 引起熟练劳动力需求的上升, 其相对工资会上升, 这样就 会激发更多的劳动者做出接受教育的选择。当跨国公司对 其分支机构雇员进行培训以及向与其配套的本地企业进行
技术咨询和培训等活动时, 就会产生对人力资本积累的溢 出效应。

此外, IFDI 还通过促进关联产业全要素生产率的提高 产生产业关联效应, 促进产业结构的升级。

\section{4. 实证检验}

为了检验IFDI 与不同技术密集度产品比较优势之间的 长期关系, 我们采用 1992-2014 年的数据, 分别用 FDI、 RCALT、RCAMT 和 RCAHT 表示按当年价格表示的外商 直接投资存量、低技术产品出口额、中技术产品出口额、 高技术产品出口额。对 FDI 的时间序列进一步用价格指数 进行平减, 为了消除物价因素的影响并消除数据中存在的 异方差, 对 FDI 取自然对数, 得时间序列数据 LNFDI。

\section{1 单位根与平稳性检验}

对 LNFDI、RCALT、RCAMT 和 RCAHT 四组数据系 列进行单位根检验, 确定其平稳性, 结果显示 (表 2), 四组数据系列均非平稳, 一阶差分后为平稳序列。

\section{2 协整检验}

将 LNFDI 分别与 $\mathrm{RCA}_{\mathrm{LT}} 、 \mathrm{RCA}_{\mathrm{MT}}$ 和 $\mathrm{RCA}_{\mathrm{HT}}$ 进行协整 检验, 检验结果如表 3 所示, LNFDI 分别与 $\mathrm{RCA}_{\mathrm{LT}} 、 \mathrm{RCA}_{\mathrm{MT}}$ 和 $\mathrm{RCA}_{\mathrm{HT}}$ 之间存在长期协整关系, 二者之间的回归不是虚 假回归。

表 2 单位根与平稳性检验

\begin{tabular}{|c|c|c|c|c|c|c|}
\hline 变量名 & & & $\mathrm{ADF}$ 值 & $1 \%$ 检验值 & $5 \%$ 检验值 & 平稳性 \\
\hline \multirow{2}{*}{$\operatorname{lnFDI}$} & \multicolumn{2}{|c|}{ 原序列 } & -2.1756 & -4.1756 & -3.6908 & 非平稳 \\
\hline & \multicolumn{2}{|c|}{ 一次差分 } & -10.3558 & -4.5716 & -3.6908 & 平稳 \\
\hline \multirow{6}{*}{$\mathrm{RCA}$} & \multirow[t]{2}{*}{$\mathrm{LT}$} & 原序列 & -1.9739 & -3.8315 & -3.0299 & 非平稳 \\
\hline & & 一次差分 & -3.3137 & -3.8574 & -3.0404 & 平稳 \\
\hline & \multirow[t]{2}{*}{ MT } & 原序列 & -1.6731 & -3.9591 & -3.0810 & 非平稳 \\
\hline & & 一次差分 & -4.3969 & -3.9591 & -3.0810 & 平稳 \\
\hline & \multirow[t]{2}{*}{$\mathrm{Ht}$} & 原序列 & -0.9474 & -3.8315 & -3.0299 & 非平稳 \\
\hline & & 一次差分 & -1.6477 & -2.6998 & -1.6066 & 平稳 \\
\hline
\end{tabular}

\section{3 长期变动关系}

构建误差修正 (ECM) 模型:

$$
\Delta R C A_{t}=\alpha_{0}+\alpha_{1} \Delta \ln F D I_{t}+E C M_{t-1}+\varepsilon_{t}
$$

其中误差修正项 $E C M_{t-1}$ 为:

$$
E C M_{t-1}=R C A_{t-1}-\beta_{0}-\beta_{1} \ln F D I_{t-1}-\varepsilon_{t-1}
$$

呈负相关关系; 而中技术和高技术的比较优势与 IFDI 呈正 相关关系, 而且 IFDI 对高技术产品比较优势的提高程度大 于对中技术产品比较优势的提高程度, IFDI 有利于提高中 国高技术密集度产品的比较优势, 有利于中国比较优势由 低端产品向高端产品转变。（见表 4) 


\section{5. 结论}

中国比较优势产品结构水平不断提高, IFDI 整体上有 利于中国比较优势产品结构的升级。IFDI 弥补了中国的资 本缺口，对低技术产品的比较优势起到了积极作用; IFDI 促进了中国技术密集型产品比较优势水平的提高, 有利于
中国比较优势产品结构升级。中国在经济发展和促进经济 结构转型的过程中, 应该继续积极吸引外商直接投资, 同 时加大对外商直接投资的产业导向力度，引导跨国公司投 资于中国的高新技术产业，促进 IFDI 的溢出效应和产业关 联效应，进一步提升中国比较优势产品的结构水平。

表 3 协整检验结果

\begin{tabular}{|c|c|c|c|c|c|}
\hline & 特征值 & 统计量 & 5\%临界值 & $1 \%$ 临界值 & 假设 No. of CE(s) \\
\hline$R C A_{L T}$ & 0.869814 & 38.62209 & 8.39771 & 3.15239 & None \\
\hline LNFDI & 0.101366 & 1.923838 & 3.841466 & 6.34897 & At most 1 \\
\hline$R C A_{M T}$ & 0.640356 & 21.46521 & 5.49471 & 19.93711 & None \\
\hline LNFDI & 0.156227 & 3.057688 & 3.841466 & 6.34897 & At most 1 \\
\hline$R C A_{H T}$ & 0.755177 & 26.68292 & 6.83035 & 23.15239 & None \\
\hline LNFDI & 0.072409 & 1.352956 & 3.841466 & 6.34897 & At most 1 \\
\hline
\end{tabular}

表 4 不同技术密集度产品比较优势与 $\ln F D I_{t}$ 的误差修正模型估计结果和长期均衡关系

\begin{tabular}{ccc}
\hline \hline 产品技术类型 & 误差修正模型估计结果 & 长期均衡关系 \\
\hline 低技术 & $\Delta R C A_{t}=-0.0284-0.0973 \Delta \ln F D I_{t}+0.4015 E C M_{t-1}$ & $R C A_{t}=5.0751-0.1992 \ln F D I_{t}$ \\
\hline 中技术 & $\Delta R C A_{t}=-0.0083+0.0610 \Delta \ln F D I_{t}+1.2469 E C M_{t-1}$ & $R C A_{t}=0.0802+0.0380 \ln F D I_{t}$ \\
\hline 高技术 & $\Delta R C A_{t}=0.0350+0.0469 \Delta \ln F D I_{t}+0.2177 E C M_{t-1}$ & $R C A_{t}=-1.7048+0.1807 \ln F D I_{t}$ \\
\hline \hline
\end{tabular}

\section{参考文献(References)}

[1] China's National Bureau of Statistics. 2013 China statistical yearbook, China statistical publishing house.

[2] JIANG Dianchun, ZHANG yu. Industrial Characteristics and Technology Spillover of FDI: The Empirical Evidence of Chinese High-tech Industries. World Economy, 2006 (10).

[3] FENG Mei. the evolution of Chinese manufacturing comparative advantage and factor endowment characteristics analysis. Statistics and Decision, 2012 (10).

[4] Balassa, B.A.. "A Stages Approach to Comparative Advantages", in Balassa. B.A.(ed), The Newly Industrializing Countries in the World Economy, New York: Pergamon Press, 1981,p.35.

[5] ZHENG Zhan-Peng. Empirical Study on foreign Trade Structure and Export Competitive of China. Journal of
International Trade , 2010 (7).

[6] Worz. J. Skill Intensity in Foreign Trade and Economic Growth. Empirica. Vol.32,No.3,2005,pp.117-144.

[7] Lall S., A, Manuel. China.s Competitive Performance: A Threat to East Asian Manufactured Exports? World Development, Vol.32, No.9, 2004b, pp.1441-1466.

[8] Technology innovation model mainly comes from the Romer (1990), Grossman and Helpman (1991), Aghion and Howitt's (1992) study.Romer, Paul M. Endogenous Technological Change. Journal of Political Economy. Vol.98, No.10, 1990, pp.71-102; Grossman G. M, E. Helpman. Quality Ladders and Product Cycles. The Quarterly Journal of Economics. Vol.106, No.2, 1991b, pp.557-586; Aghion, Philippe, Peter Howitt. A Model of Growth Through Creative Destruction. Econometrica. Vol.60,No.5.1992,pp.323-351. 